\title{
Note on Names, Terms, and Romanization
}

This book uses the pinyin romanization system for Chinese words and names, with certain exceptions. In Taiwan, many people and places (cities, for instance, though not usually neighborhoods) have their own preferred or published romanization, which takes precedence here. For a few proper nouns, customary spellings like Chiang Kai-shek and Kuomintang are employed.

Personal names and place names cited from newspapers, official records, and other public sources are real. Pseudonyms (always in pinyin) are used for the names of neighborhood research sites and for individuals referenced in accounts drawn from interviews and site visits. The handful of exceptions, in which real names are used by permission, is flagged in the notes.

Administratively speaking, Beijing covers a sprawling territorial expanse, approaching the size of New Jersey in area, including sixteen districts and two counties. This book focuses on the eight districts that constitute its urban core. Except where noted, Taipei here refers entirely to the capital city (Taibei shi), not to the separate region that surrounds it, formerly known as Taipei County and now called New Taipei City (Xinbei shi).

There is no single, standard translation for lizhang, Taiwan's official neighborhood leaders. I refer to them as neighborhood wardens (NW), and sometimes with the untranslated term. In some English-language documents they are called borough chiefs or borough wardens. This seems inapt, however, because where the term "borough" is used in large cities such as London, Montreal, and New York, it denotes an area much larger than a typical $l i$. For the sake of brevity I have left untranslated the term liganshi, referring to the civil servants sent by district offices to work with wardens, although "neighborhood officer" or "neighborhood liaison" would suffice. China's jumin 
weiyuanbui have long been straightforwardly translated as residents' committees (RC). In Beijing and elsewhere, nomenclature has evolved over the past twelve years or so, as these bodies have gradually been relabeled as community residents' committees and made into one component of larger offices called communities (shequ). Chapter 2 discusses the terminological and institutional nuances. For the sake of simplicity, this book refers to them generically as residents' committees, except in places where the precise organizational details are relevant. 
ROOTS OF THE STATE 
